\title{
One Simple Virtual Avatar System Based on Single Image
}

\author{
Lanfang Dong ${ }^{1(\bowtie)}$, Jianfu Wang ${ }^{1}, \mathrm{Kui} \mathrm{Ni}^{1}$, Yatao Wang ${ }^{1}$, Xian $\mathrm{Wu}^{1}$, \\ and Mingxue $\mathrm{Xu}^{2}$ \\ 1 School of Computer Science and Technology, \\ University of Science and Technology of China, Hefei, China \\ lfdong@ustc.edu.cn \\ $\{$ wangjf55, nique, ythwang, wuxian\}@mail.ustc.edu.cn \\ 2 College of Geophysics and Information Engineering, \\ China University of Petroleum (Beijing), Bejing, China \\ xumingxue2008@yeah.net
}

\begin{abstract}
To establish virtual avatar systems at the computing environments with limited resources, we design such a system based on single image which can generate speech animation with different facial expressions. Firstly, facial feature points are extracted automatically or manually based on MPEG-4 facial animation to build the face model. Then, according to Facial Animation Parameters of visual phoneme and expression stream, the feature points after image warping are calculated. The next step is to generate the in-between frames by using the image warping algorithm based on scan lines and combining with the correlative time parameters. Finally, with adding the actions such as nodding, shaking heads and winking, the face animation is implemented. Experimental results show that the proposed system can generate smooth and realtime facial expressions under platforms with low computing capacity.
\end{abstract}

Keywords: MPEG-4 · Image warping · Face animation · Visual phoneme

\section{Introduction}

As the speedy development of mobile communication technology and the rapid popularization of mobile network, the mobile devices such as Smartphone, PAD are widely used. However, they are also facing increasing challenges from high power consumption. Limited to the actual objective conditions, the mobile devices are often unable to get power supply in time. Therefore, providing the low power software for platforms with limited computing capacity has become one of the main solutions and also become one of the focuses in software development.

Combining with computer graphics, computer animation and virtual reality, the virtual avatar technology is used widely at present. Especially along with the hot showing of science fiction movie, Avatar, this technology is known by more people, and is employed by more animation and movie production companies. 
However, one of key problems in virtual avatar is that how to provide realistic and realtime animation in the platforms with limited computing capacity.

Designing a virtual avatar system generating natural and realtime face expressions with low power consumption is one focus of the recent research. In this paper, we design one simple virtual avatar system which is implemented based on single image and can achieve accurate and realtime fusion and synchronization of facial expressions, mouth shapes and voice. Besides, the system adopts one image warping algorithm based scan lines to avoid high power consumption on the mobile devices.

\section{Related Work}

Facial expressions and speech are two most important communication ways for human, as well as the key parts in virtual avatar system. How to implement the fusion and synchronization of facial expressions, mouth shapes and voice accurately, realistically and in real time is presently one hot research in computer animation and virtual reality.

Nowadays 3D facial animation is studied widely and deeply. The first step is usually to create the $3 \mathrm{D}$ face model [1], and then different approaches are employed to control the face motion. The methods for 3D facial animation can divided into two categories: the parameter model based technology and physical model based technology [2]. Parke [3] firstly introduced the parameterized models for facial animation, and with controlling the expression parameters and structural parameters to synthesize different facial expressions and postures, but the reality remained to be improved. Waters [4] presented a muscle model based on the physical properties of muscle, which can create the subtle expressions using muscle vectors and radial functions. The difficulty of this method is to master the anatomy, histology and dynamics of facial muscles. The Facial Animation Parameters (FAP) in MPEG-4 standard [5] defines a complete collection of basic movements which can be used to generate facial expressions. Currently, the MPEG-4 based methods are widely used for 3D facial animation [6-9]. Physics-based facial animation typically use mass-spring or finite element networks to model the elastic properties of skin [10,11]. In paper [12], one physical 3D facial model consisting of cutaneous tissue, subcutaneous fatty tissue, and muscles is embedded in the mass spring system to generate realistic facial animation. The other methods based on physical model [13-15] can enhanced the reality of animation. However, one general shortcoming of $3 \mathrm{D}$ facial animation is high computational complexity. In addition, the modeling of 3D faces usually needs special equipment of high construction cost. Another way for facial animation is based on images. Because the textures come directly from the images, images based method can provide facial expressions with high reality. Arad et al. [16] proposed one image morphing algorithm with Radial Basis Function (RBF) to generate facial animation based on single image. The facial animation based on key frames is one of the most widely used methods in computer animation [17]. This method was first put forward by Beier and Neely [18], and in this 
method corresponding triangular meshes with similar topological structures are constructed for the neighboring key frames. Afterward, more key-frame based methods are proposed to improve the animation effects [19,20]. However, all those approaches with neglecting the fusion of mouth shapes and facial expressions are not suitable for facial animation driven by speech.

In addition, current speech animation systems are mostly concerned with generating the lip animation to match the speech and missing the face expressions, while facial animation mainly focuses on modeling of the face and the facial motion analysis. In paper [21] motion capture-based facial animation extracted information from the expression axis of a speech performance. To map the audio information to the visual information representing the facial movements, paper [22] trained a set of neural networks using a large collected set of audioCvisual training data from real human subjects. Similarly, in paper [23] a novel motion capture mining technique was provided with learning speech coarticulation models and expression spaces from recorded facial motion capture data. To generate one lively and convincing animation, sophisticated facial motions are required in motion capture-based algorithms. Above all, on the one hand, the current researches cannot balance the facial expressions, lip movements and speech. On the other hand, the high computational complexity brought by the animation limits the use of corresponding software or systems in mobile devices. Different from the previous complex spline interpolation, 3D model-based or motion capture-based approaches with sophisticated facial motions, in this paper the proposed virtual avatar system is implemented on single image with simple algorithm and low power consumption. The system employs the image warping based on scan lines to accomplish the facial animation quickly and effectively with less computing resource and memory space. Moreover the system adds some other actions, such as nodding and winking, and it can also be used to generate the facial animation of animals and cartoon characters.

\section{Image Warping Based on Scan Lines}

Our proposed simple virtual avatar system is designed based on single image, and the key is implementing of the image warping. Different from the prior image warping based on scattered points or fragments, in this paper we use the image warping algorithm proposed in [24]. To make it simple, the main task is to generate the warped target image using the input source image and the known feature points in both source and target images. The detailed process is illustrated as follows:

Firstly, the facial feature points are defined as shown in Fig. 1, and the next section offers specific details on feature points.

Secondly, neighboring feature points are joined by splines as Fig. 2 shows, and the points located in the splines are calculated by liner interpolation. Some other points are added to limit movement range of feature points distributed within the rectangular boundaries as shown in Fig. 2. The addition of those points is helpful for the control of face movement and the construction of splines. 


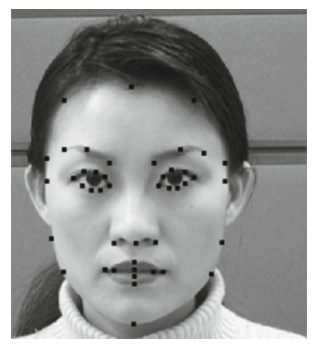

Fig. 1. Facial feature points.

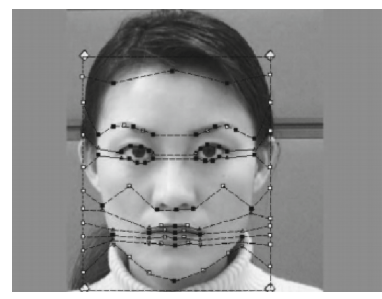

(a)

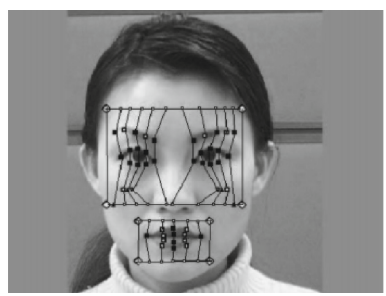

(b)

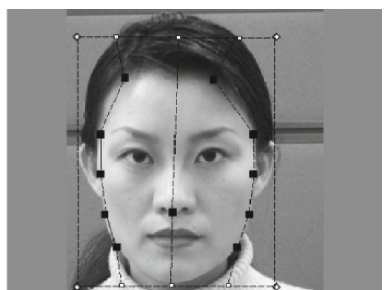

(c)

Fig. 2. Facial splines (a) Horizontal facial splines (b) Vertical facial splines (c) Vertical splines of face contour.

The splines consist of horizontal splines and vertical splines, of which the horizontal splines covering the whole face are used to control the face warping in vertical direction, as Fig. 2(a) shows, vertical splines covering the mouth and eye areas are used for horizontal warping of mouth and eyes, as Fig. 2(b) shows, while the vertical splines of face contour are used to control the shaking of head as shown in Fig. 2(c). The horizontal splines and vertical splines are constructed independently without sharing the same feature points to avoid generating spline grid and can be used in the following image warping separately.

Here, we set the images before and after warping as the source image and target image separately. Then, after the construction of facial splines in both two images, the coordinates of points between splines from the source image to the target are calculated, of which the $y$ coordinates are calculated according the horizontal splines of two images while the $x$ coordinates are calculated according the vertical splines. In this step, the scan lines are used as shown in Fig. 3, where Fig. 3(a) shows the vertical displacement while Fig. 3(b) shows the horizontal displacement.

We take the calculation of vertical displacement of one point $C$ as an example. The solid splines in Fig. 3(a) separately represent the horizontal spline1 and spline 2 in source image while the dotted splines separately represent the horizontal spline $1^{\prime}$ and spline $2^{\prime}$ in target image. The vertical line is one vertical scan line which progressively scans the horizontal splines in source and target images. In Fig. 3(a), $A$ is the intersection of scan line and spline 1 while $B$ is the 


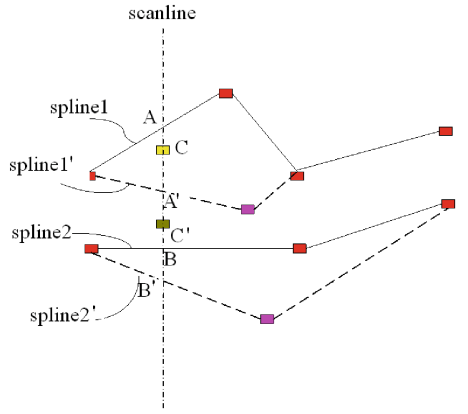

(a)

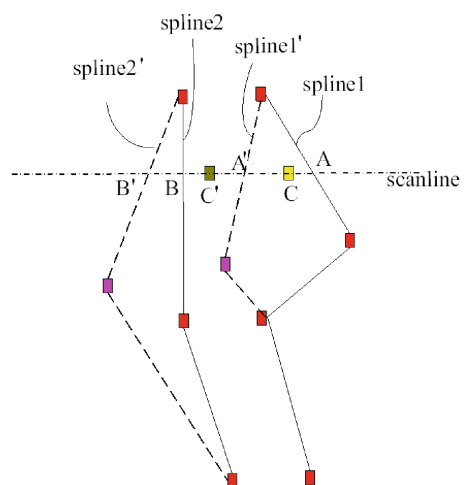

(b)

Fig. 3. (a) scan line between horizontal splines (b) scan line between vertical splines.

intersection of scan line and spline 2 . Their corresponding intersections in target image are $A^{\prime}$ and $B^{\prime}$. $C$ is one point located in scan line between $A$ and $B$ in source image, and its corresponding point in target image is $C^{\prime}$.

Assuming $A(x, s 1), B(x, s 2), A^{\prime}(x, d 1), B^{\prime}(x, d 2), C(x, y), C^{\prime}\left(x, y^{\prime}\right)$, the vertical coordinate $y^{\prime}$ of $C^{\prime}\left(x, y^{\prime}\right)$ is calculated by linear interpolation formulation as (1).

$$
y^{\prime}=d 1+(y-s 1) \times \frac{(d 2-d 1)}{(s 2-s 1)}
$$

The vertical displacement from $C$ to $C^{\prime}$ is $\Delta y=y^{\prime}-y$. Similarly, the horizontal displacement $\Delta x$ of $C$ can be achieved by using the scan lines between the vertical splines, as shown in Fig. 3(b).

In this way, we can get the coordinate of $C^{\prime}(x+\Delta x, y+\Delta y)$ after warping. Finally, when coordinates of all points are achieved, the warping image, namely the target images, is generated based on the feature points.

\section{Virtual Avatar System}

The proposed virtual avatar system consists of three parts: facial modeling, speech processing and facial expression processing.

\subsection{Facial Modeling}

The first step of virtual avatar is to build parametric model of the face and then to define and mark the facial feature points on the model. MPEG-4 provides the face mode standard and corresponding face definition parameters (FDP). To build one nice face model, the feature points should be representative, express the face features and be able to uniquely identify the face. In this paper, the feature points we selected from FDPs are shown in Fig. 4, including mouth, eyes, brows, nose and the face contour. 


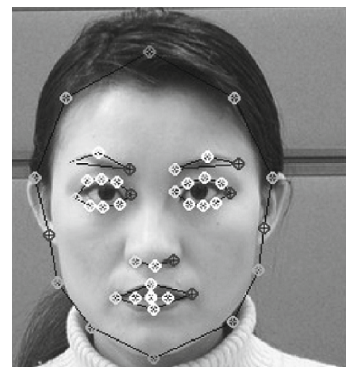

(a)

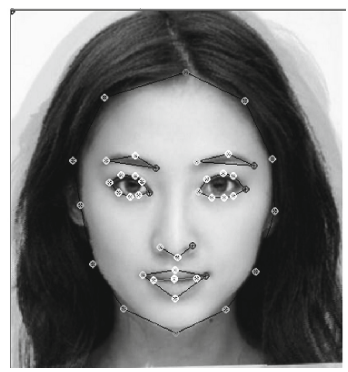

(b)

Fig. 4. The feature points of face model.

\subsection{Speech Processing}

In this stage, the input audio files are transformed by speech recognition engine to produce phoneme-timestamp streams, which are further transformed to visual phoneme and timestamp streams, through which the locations of facial feature points are achieved. Based on the feature points, image warping is implemented to generate the facial animation. At last the synchronization of speech and animation is achieved according to the timestamps of phonemes. In this system, we use Microsoft Speech Recognizer as the speech recognition engine to convert the one audio to the phoneme streams with timestamp.

Visual Phoneme. Speech utterance can be segmented into sentences, words, or even phonemes, where phoneme is the smallest unit. The phoneme- timestamp streams reflect the duration of one phoneme. According to MPEG-4, the phonemes with the same lip movements are classified together as one visual phoneme. Each visual phoneme is corresponding to a set of FAPs, where Table 1 shows one sample. MPEG-4 provides more detailed visual phonemes and FAPs.

As Table 1 shows, the visual phoneme has a corresponding set of masks and maximum values for FAPs. In this table, ae, $a x$, ah, aa and ay belong to one visual phoneme with the same lip movements when speaking. The following number 0 is the label of the visual phoneme. There are 68 FAPs in MPEG-4. The next 68 numbers ( 0 or 1 ) are the masks of FAPs where 0 illustrates the FAP does not work while 1 works. The numbers in last line are the coefficients of FAPs with corresponding masks equal to 1 , where absolute value is the magnitude of displacement while the sign is the direction of displacement.

After the locations of feature points are calculated based on the visual phonemes, we use the scan-line based image warping algorithm to get the image after warping. The frames between images before and after warping are generated by simple linear interpolation.

The relationship of facial expressions and FAPs is same as the visual phonemes with referencing to MPEG-4 standard. The MPEG-4 Facial Animation Standard is simplified and applied in this 2D facial animation generating. 
Table 1. One sample of visual phoneme

\begin{tabular}{|c|c|c|c|c|c|c|c|c|c|c|c|c|c|c|c|}
\hline$\#$ & ae & $a x$ & $\mathrm{ah}$ & aa & ay & 0 & & & & & & & & & \\
\hline 0 & 0 & 1 & 1 & 1 & 1 & 1 & 0 & 0 & 1 & 1 & 0 & 0 & 0 & 0 & 0 \\
\hline 0 & 0 & 0 & 0 & 0 & 0 & 0 & 0 & 0 & 0 & 0 & 0 & 0 & 0 & 0 & 0 \\
\hline 0 & 0 & 0 & 0 & 0 & 0 & 0 & 0 & 0 & 0 & 0 & 0 & 0 & 1 & 0 & 1 \\
\hline 0 & 0 & 0 & 0 & 1 & 1 & 1 & 1 & 0 & 0 & 1 & 1 & 0 & 0 & 0 & 0 \\
\hline 0 & 0 & 0 & 0 & 0 & 0 & & & & & & & & & & \\
\hline 180 & 60 & -350 & 30 & 0 & 30 & 0 & 0 & -407 & 30 & 30 & 0 & 0 & & & \\
\hline
\end{tabular}

Transition of Visual Phonemes. If the shape of mouth recovers to neutral state every time when the process of each visual phoneme is finished, the generated facial expressions will be unrealistic. Therefore, to remedy abnormal expressions, we introduced the transition of visual phonemes based on the linear interpolation method. New FAPs are interpolated based on prior FAPs and current FAPs to generate new expressions to instead the nature state. The experimental results show that the introduction of transition can achieve the reality and realtime requirement. Figure 5(a) shows movement amplitudes without transition while Fig. 5(b) shows amplitudes with transition.

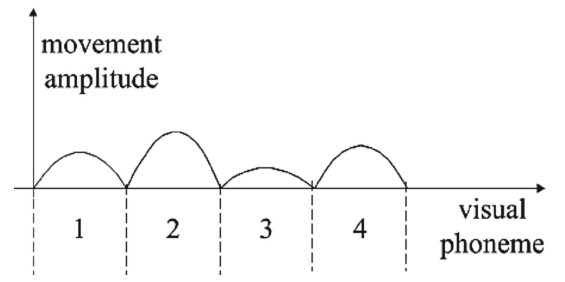

(a)

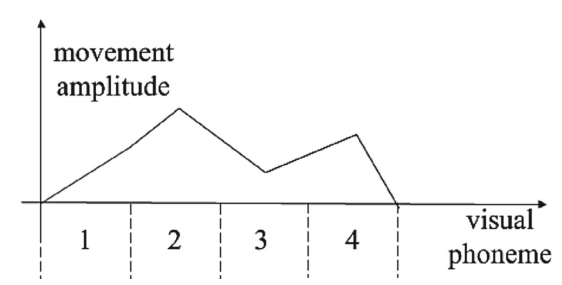

(b)

Fig. 5. (a) The movement amplitudes of visual phonemes without transition (b) The movement amplitudes of visual phonemes with transition.

Merging of Visual Phonemes. If the frame rate is $20 \mathrm{fps}$ while the lasting time of visual phonemes is too short to produce one frame, some abnormal expressions such as sudden change of mouth will emerge. Therefore, the visual phonemes with short duration should be merged to create more fluent and natural animation in real time as Fig. 6 shows.

\subsection{Expression Processing}

To make the generated animation more realistic and natural, our designed system takes both lip movements and facial expressions into consideration. In addition, we add some other random face motions, such as nodding, shaking heads and winking to generate more abundant facial animation. 


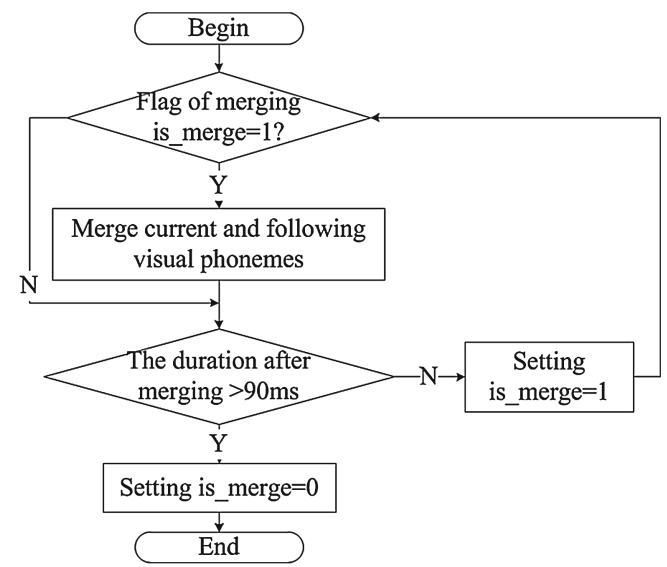

Fig. 6. The merging of visual phonemes.

Facial Expression. The relationship of facial expressions and FAPs is similar to the visual phoneme, so do the transition and merging of parameters. With considering the lip movements and facial expressions, our system completes the fusion of FAPs of visual phonemes and facial expressions. The fusion is to put the FAPs of visual phonemes and FAPs of facial expressions together, which brings synchronization of lip movements and facial expressions at the same time. Specifically, the fusion of FAPs is achieved by using the Boolean OR operator for corresponding masks and arithmetic operation Addition for coefficients of visual phonemes and facial expressions.

According to the parameters after fusion, the locations of facial feature points are achieved, which are further used to warp the face image, where the image warping algorithm is based on the scan-line method.

Winking. In this part, we add the winking to the facial expressions using four FAPs concerning with the eyes: both upper and lower eyelids of eyes, which are also used in FAPs of facial expressions. Therefore, we set the winking as an individual expression. When winking occurs, the other FAPs related to eyes will be disabled. The process of winking in the virtual avatar system is shown in Fig. 7.

Head Movements. The head movements consist of shaking head and nodding, which are set as periodic movements in this paper, and periodic changes of motion amplitudes are shown in Fig. 8.

The nodding is implemented by adding same value to the $\mathrm{Y}$ value of all feature points while the shaking head is moving the vertical splines of face contour as shown in Fig. 2(c) where the moving amplitude of the middle spline is larger than the others. Figure 9 shows the process of nodding, and the shaking has similar process. 


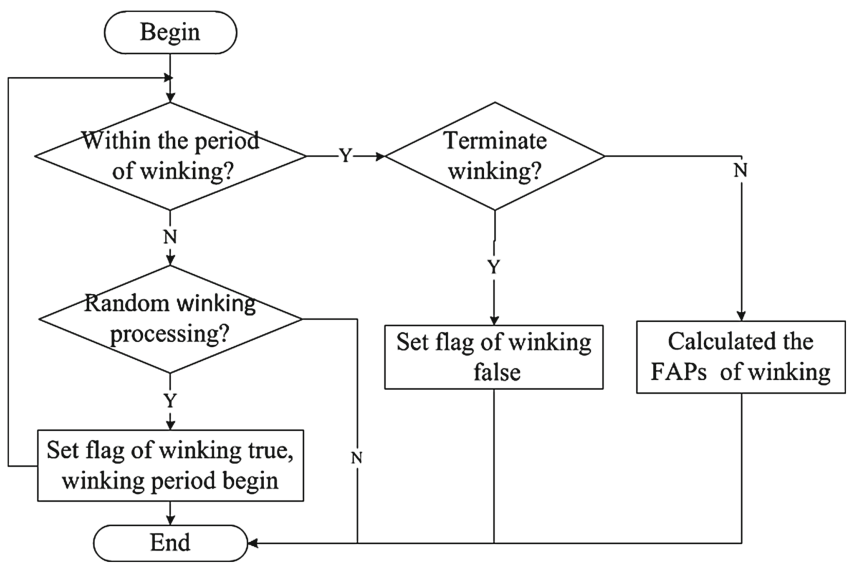

Fig. 7. Process of winking.

Shading of Mouth. Under the nature state, the lips are pressed together. When speaking, the shapes of mouth change with the opening and closing of mouth alternately. When the mouth is opening, the region lies between two lips should be shaded. Figure 10 shows the shading process of mouth. The inner lips can be seen as two curves, which are computed at the first step of shading.

For the convenience of computing, in the system the feature points of mouth are rotated to horizontal direction firstly, and then we use Lagrange interpolation to obtain the inner boundary curves of mouth. To simplify the shading of mouth and ensure the quality of generated animation, we combined the sample image of mouth into the neutral face image so that the inner structure of mouth could be shown when mouth was open. In facial animation, the warping of mouth is based on it center. Within the mosaic and fusion of mouth sample image and face image, the corresponding center points first are matched. Then the mouth sample image is rotate to the same direction as face image and its inner lip curves is also get. According to the corresponding points in the curves, the sample image is scaled and warped to suit change of mouth in face image which can be achieved with simple linear interpolation.

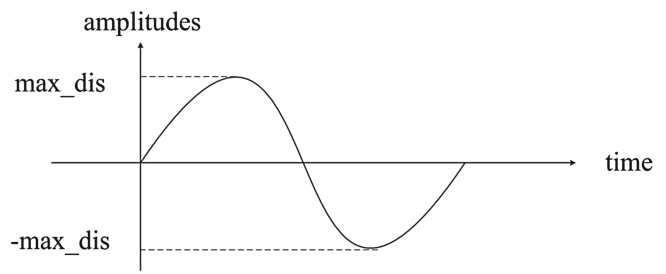

Fig. 8. Periodic changes of amplitudes. 


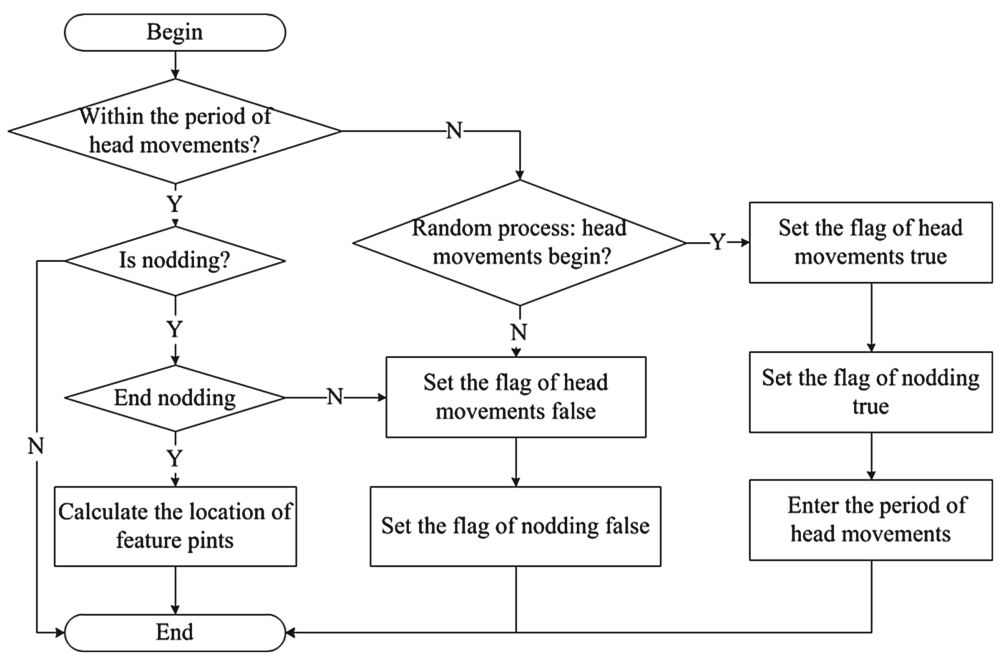

Fig. 9. The process of nodding.

\subsection{The Virtual Avatar System}

After the facial modeling, speech processing and expression processing, we get the whole realization process as shown in Fig. 11, where the upper dotted box presents the calculation of displacement of feature points while the lower is the corresponding image warping. The additional points are shown as Fig. 2(a)-(c), located on the sides of rectangles.

\section{Experiments and Discussion}

\subsection{Experimental Results}

Figures 12, 13, 14, 15 and 16 show the samples of generated animation by the virtual avatar system. The Figs. 12, 13 and 14 are the facial animation generated from three different human face images, from which we can see the facial expressions of delight (as Figs. 12(a) and 13(a)), anger (as Figs. 12(b) and 13(b)) and surprise (as Figs. 12(c) and 13(c)) respectively and corresponding mouth movements. The system can also be used to generate the facial animation of cartoon characters and animals as shown in Figs. 15 and 16. From the figures, we can see the winking as Figs. 15(c) and 16(c) show, and the motions of head as Figs. 16(a) and 16(c) show.

The action of the brows, eyes and mouth can best reflect the facial expression. When the emotions of one person or animal change, the shape information of such organs changes correspondingly. As Fig. 15 shows, the shapes of the brows, eyes and mouth are in harmony with emotion. In terms of animation effects, the generated facial expressions for cartoon characters and animals are better than that for humans. From the Figs. 13 and 14, some distortion occurred when the 


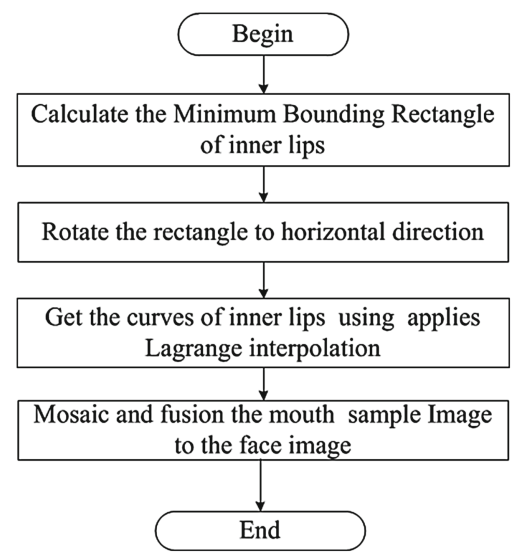

Fig. 10. The shading of mouth.

eyes were closed. On the contrary, the action of wink for cartoon and animals looks more natural.

It is also worth mentioning that the shading of mouth can help to promote reality. Such as Figs. 13, 14(c), 16(b) and 16(c) when the mouth is open widely, the teeth look natural. The results of the experiment clearly demonstrate that the system can generate realistic facial animation with both mouth movements and facial expressions.

\subsection{Discussion}

Compared with the current facial animation achieved from multiple images, videos $3 \mathrm{D}$ models, such as the realtime facial animation based on 3D models in $[25,26]$, the approach in this paper with only one available image cannot show better animation effect. However, the proposed system enlarges the application fields of animation. On the other hand, it also contains advantages of storage, computation and communication. Different from previous methods, the proposed virtual avatar system is implemented based on single image, and every frame of the facial animation is generated with only using the input image, FAPs and the essential feature points, so that this system needs small computer memory and storage space. Within the communication of different mobile devices, only facial feature points are transferred after the completion of facial modeling to produce the warping images. Furthermore, the key scan-line based algorithm of this system can achieve the warping image with the simplest linear interpolation without complex calculations, which has the advantages of saving computation time and computing resources and avoids higher power consumption. Another advantage of scan-line based algorithm is that the interpolation points can controlled within the feature points, so are the corresponding values. Therefore, the abnormal warping images and the boundary overstepping can be avoided so that the quality of generated animation can be improved further. In a word, the 


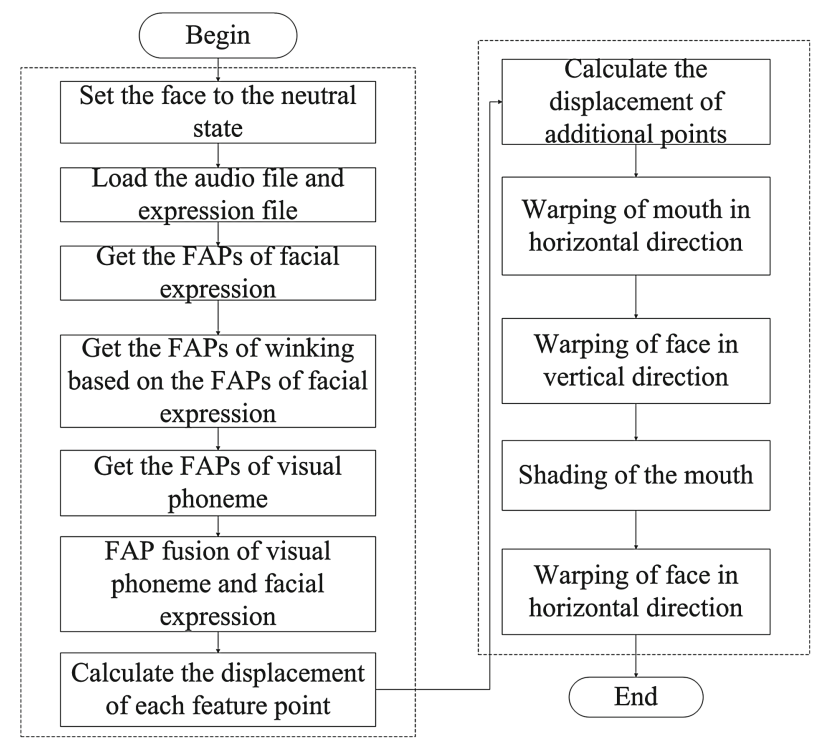

Fig. 11. The whole realization process of the virtual avatar system.

proposed avatar system can provide facial animation of high quality and with effectively saving the computing cost.

However, according to the experimental results, some details of human facial expressions need to be refined. For the human face, the feature points can be extracted by the system automatically. But for cartoon characters and animals, the feature points location is achieved manually. Therefore, the presented avatar system should be improved in the effect of human facial expression animation and the self-adaptive operation.

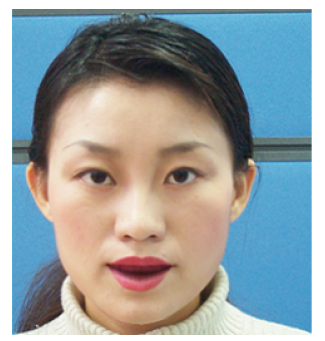

(a)

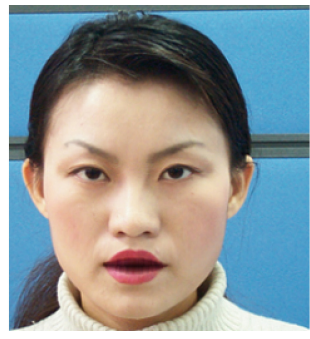

(b)

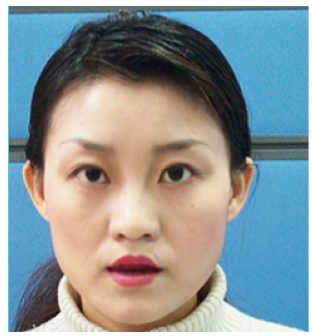

(c)

Fig. 12. The first sample of human facial animation. 


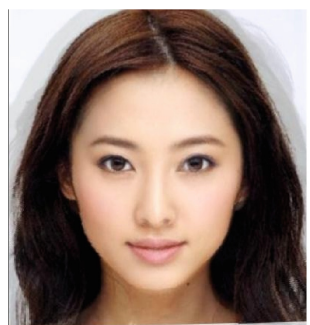

(a)

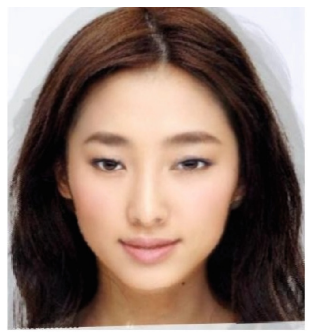

(b)

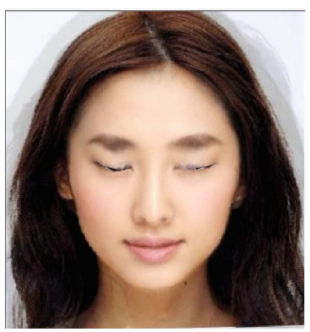

(c)

Fig. 13. The second sample of human facial animation.

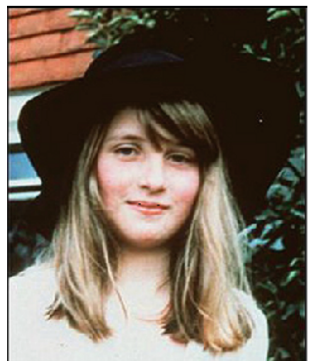

(a)

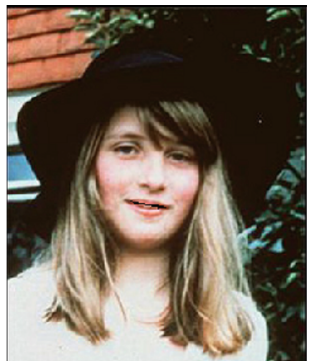

(b)

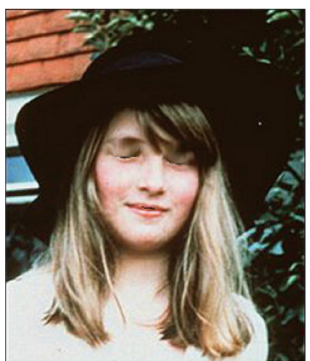

(c)

Fig. 14. The third sample of human facial animation.

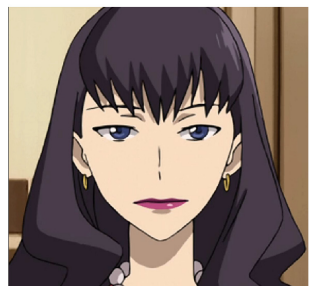

(a)

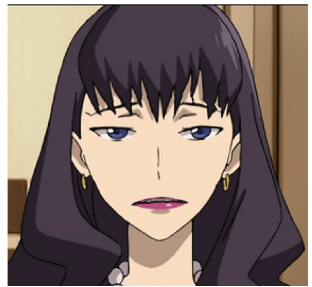

(b)

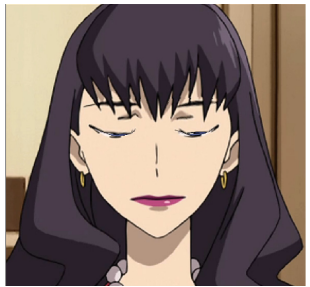

(c)

Fig. 15. The sample of facial animation for cartoon character.

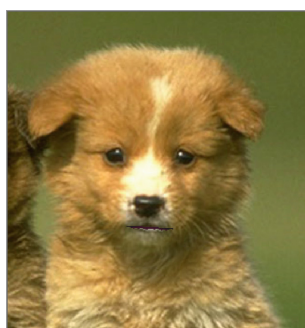

(a)

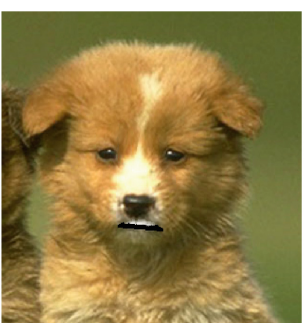

(b)

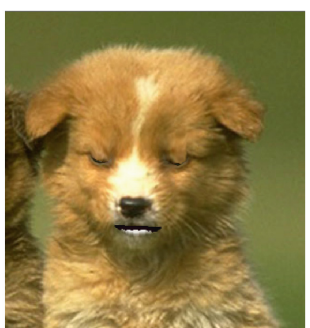

(c)

Fig. 16. The sample of animal facial animation. 


\section{Conclusions}

This paper provided one simple virtual avatar system based on single image, which generated the facial animation with fusing the facial expressions and synchronization of mouth movements and speech. In addition, the system is implemented using the image warping algorithm based on scan lines that brings less calculation and high velocity, and we can run our system on the normal computers in real time. To make the animation more abundant and more realistic, some actions such as nodding, shaking heads and winking are introduced. The system can also be used to generate the facial animation of cartoon characters and animals. The experimental results showed that the proposed virtual avatar system is of excellent performance and practical value.

\section{References}

1. Lee, Y., Terzopoulos, D., Waters, K.: Realistic modeling for facial animation. In: ACM Proceedings of the 22nd Annual Conference on Computer Graphics and Interactive Techniques, pp. 55-62. ACM Press, New York (1995)

2. Kahler, K., Haber, J., Seidel, H.P.: Geometry-based muscle modeling for facial animation. In: Graphics Interface, pp. 37-46. Morgan Kaufmann Press, Toronto (2001)

3. Parke, F.I., Waters, K., Alley, T.R.: Computer Facial Animation. AK Peters Wellesley, Mass (1996)

4. Waters, K.: A muscle model for animation three-dimensional facial expression. ACM SIGGRAPH Comput. Graph. 21, 17-24 (1987)

5. Pandzic, I.S., Forchheimer, R.: MPEG-4 Facial Animation: The Standard, Implementation and Applications. Wiley, New York (2002)

6. Duarte, R.L.P., Rhalibi, A.E., Merabti, M.: Coarticulation and speech synchronization in MPEG-4 based facial animation. Kybernetes: Int. J. Syst. Cybern. 43, 1165-1182 (2014)

7. Parreira, D.R., El, R.P., Carter, C., Merabti, M.: Survey and evaluation of Mpeg-4 based 3D character animation frameworks. In: 2013 5th International Conference on Games and Virtual Worlds for Serious Applications, pp. 1-8. IEEE Press, Dorset (2013)

8. Obaid, M., Mukundan, R., Billinghurst, M., Pelachaud, C.: Expressive MPEG-4 facial animation using quadratic deformation models. In: 2010 Seventh International Conference on Computer Graphics, Imaging and Visualization, pp. 9-14. IEEE Press, Sydney (2010)

9. El Rhalibi, A., Carter, C., Cooper, S., Merabti, M.: Highly realistic MPEG-4 compliant facial animation with charisma. In: 2011 Proceedings of 20th International Conference on Computer Communications and Networks, pp. 1-6. IEEE Press, Lahaina (2011)

10. Lee, Y., Terzopoulos, D., Waters, K.: Constructing Physics-based Facial Models of Individuals. Graphics Interface, Canadian Information Processing Society (1993)

11. Koch, R.M., Gross, M.H., Bosshard, A.A.: Emotion editing using finite elements. Comput. Graph. Forum 17, 295-302 (1998). Blackwell Publishers Ltd

12. Anderson, R., Stenger, B., Wan, V., Cipolla, R.: Expressive visual text-to-speech using active appearance models. In: 2013 IEEE Conference on Computer Vision and Pattern Recognition (CVPR), pp. 3382-3389, IEEE Press, Portland (2013) 
13. Li, D., Sun, C., Hu, F., Zang, D., Wang, L., Zhang, M.: Real-time performancedriven facial animation with 3ds Max and Kinect. In: 2013 3rd International Conference on Consumer Electronics, Communications and Networks, pp. 473-476. IEEE Press, Xianning (2013)

14. Yu, H., Garrod, O., Jack, R., Schyns, P.: Realistic facial animation generation based on facial expression mapping. In: Fifth International Conference on Graphic and Image Processing, pp. 906903-1-906903-5. SPIE Press, Hong Kong (2014)

15. Zhang, Y., Prakash, E.C., Sung, E.: A new physical model with multilayer architecture for facial expression animation using dynamic adaptive mesh. IEEE Trans. Vis. Comput. Graph. 10, 339-352 (2004)

16. Arad, N., Dyn, N., Reisfeld, D., Yeshurun, Y.: Image warping by radial basis functions: application to facial expressions. Graph. Models Image Process. 56, 161-172 (1994)

17. Pan, H., Liu, Y., Xu, G.: Review on methods of facial synthesis. Appl. Res. Comput. 25, 327-331 (2008)

18. Beier, T., Neely, S.: Feature-based image metamorphosis. ACM SIGGRAPH Comput. Graph. 26, 35-42 (1992)

19. Edge, J.D., Maddock, S.: Image-based talking heads using radial basis functions. In: IEEE Proceedings of Theory and Practice of Computer Graphics, pp. 74-80. IEEE Press, Birminghan (2003)

20. Perng, W.L, Wu, Y., Ouhyoung, M.: Image talk: a real time synthetic talking head using one single image with chinese text-to-speech capability. In: Pacific Graphics' Sixth Pacific Conference on Computer Graphics and Applications, pp. 140-148. IEEE Press, Singapore (1998)

21. Chuang, E., Bregler, C.: Mood swings: expressive speech animation. ACM Trans. Graph. 24, 331-347 (2005)

22. Hong, P., Wen, Z., Huang, T.S.: Real-time speech-driven face animation with expressions using neural networks. IEEE Trans. Neural Netw. 13, 916-927 (2002)

23. Deng, Z., Neumann, U., Lewis, J.P., Kim, T.Y., Bulut, M., Narayanan, S.: Expressive facial animation synthesis by learning speech coarticulation and expression spaces. IEEE Trans. Vis. Comput. Graph. 12, 1523-1534 (2006)

24. Dong, L., Wang, Y., Ni, K., Lu, K.: Facial animation system based on image warping algorithm. In: 2011 IEEE International Conference on Electronics, Communications and Control, pp. 2648-2653. IEEE Press, Ningbo (2011)

25. Weise, T., Bouaziz, S., Li, H., Pauly, M.: Realtime performance-based facial animation. ACM Trans. Graph. 30, 77-86 (2011)

26. Bouaziz, S., Wang, Y., Pauly, M.: Online modeling for realtime facial animation. ACM Trans. Graph. 32, 40-49 (2013) 